\title{
Von der Resektionsprothetik zur freien Knochenplastik
}

\author{
Voraussetzungen zur Schaffung eines Knochen-Transplantationskonzeptes \\ am Unterkiefer \\ von Guido Sigron
}

ZuSAMMENFASSUNG

Die vorliegende Arbeit untersucht die Entwicklung zahnärztlicher und chirurgischer Methoden zur Wiederherstellung der Unterkieferkontinuität nach Knochenverlust.

Ausgehend von der zahnärztlichen Resektionsprothetik werden wichtige Etappen zur Knochentransplantation am Unterkiefer aufgezeigt. Bis 1914 konnte kein sicheres Transplantationskonzept erarbeitet werden. Erst die Zusammenarbeit zwischen Chirurgen und Zahnärzten im Ersten Weltkrieg schuf die Grundlagen für den Durchbruch der Knochenplastik am Unterkiefer.

\section{Einleitung}

Die Entwicklung planmässiger Kieferresektionen zählt zu den Fortschritten der Chirurgie des 19. Jahrhunderts. Die erste Teilresektion zur Entfernung eines Unterkiefersarkoms nahm 1812 der französische Chirurg Guillaume Baron de Dupuytren vor ${ }^{1,2} .1821$ dehnte der deutsche Chirurg Carl Ferdinand von Graefe die Teilresektion zu einer einseitigen Exartikulation des Unterkiefers aus ${ }^{3}$. Erst 1843 wagte der italienische Chirurg Bartolomeo Signorini die Totalexstirpation des Unterkiefers ${ }^{4}$.

Anlass zu diesen Resektionen gaben vor allem Geschwülste, die vor dem 19. Jahrhundert nicht operativ behandelt wurden. Erst der Durchbruch der lokalistisch-pathologischen Anatomie in der Chirurgie des 19. Jahrhunderts forderte auch die generelle Entfernung der Kiefergeschwülste. Ab 1830 widmete jedes umfassende Chirurgielehrbuch einen Abschnitt den Kieferresektionen. Darin wurden die verschiedenen chirurgischen Methoden und die damit verbundenen Komplikationsmöglichkeiten erklärt. Eine prothetische Nachbehandlung wurde nur für den Oberkieferdefekt erwähnt ${ }^{5,6,7}$. 
In dieser Arbeit soll abgeklärt werden, welche chirurgischen und zahnärztlichen Massnahmen zur Versorgung eines Knochenverlustes am Unterkiefer notwendig waren.

Zur Untersuchung gelangt der Zeitraum zwischen 1850 und 1918. Dieser Zeitabschnitt ist quellenmässig gut belegt. Neben chirurgischen und zahnärztlichen Lehrbüchern gibt es einige Fachzeitschriften, deren Umfang zwischen 1914 und 1918 stark ansteigt.

In der vorhandenen Literatur zur Zahnmedizingeschichte wird auf die Entwicklung chirurgischer und zahnärztlicher Methoden zur Überbrückung eines Knochenverlustes im Unterkiefer vor dem 20. Jahrhundert nicht eingegangen. Hingegen gibt es über den Zeitabschnitt zwischen 1900 und 1918 drei Arbeiten von Walter Hoffmann-Axthelm, die einen guten Überblick über die Entwicklung chirurgischer Methoden zur Knochentransplantation vermitteln ${ }^{8}$. In Hoffmann-Axthelms Arbeit «Zur geschichtlichen Entwicklung des Knochenersatzes im Unterkiefer» werden auch kurz die chirurgischen Methoden aus dem Ersten Weltkrieg besprochen, wobei der zahnärztliche Beitrag zur Knochentransplantation unerwähnt bleibt. Eine andere Arbeit, die sich auch mit Rekonstruktionsmethoden aus dem Ersten Weltkrieg beschäftigt, stammt von Roland Schmocker. Er vergleicht die Wiederherstellungsmethoden aus dem Ersten Weltkrieg mit den heute gültigen Kriterien der Knochenchirurgie ${ }^{9}$.

Um die erwähnte Fragestellung zu untersuchen, wird in 3 Schritten vorgegangen :

1. sollen wichtige Aspekte der Resektionsprothetik aufgezeigt und analysiert werden.

2. soll ein Einblick in die Erforschung der Transplantationsmechanismen und deren Auswirkung auf die skelettale Unterkieferrekonstruktion vermittelt werden und

3. wird anhand des deutschen Beispieles der Einfluss des Ersten Weltkrieges auf die Entwicklung der Knochentransplantation am Unterkiefer untersucht.

\section{Die Entwicklung der Resektionsprothetik}

Die prothetische Versorgung eines Unterkiefer-Resektionsdefektes setzt im Vergleich zum Zeitpunkt der ersten Resektion von Dupuytren - spät ein. Die Ursache dafür war das Fehlen eines plastisch verformbaren und danach 
aushärtbaren Materials. Erst mit der Entdeckung der Vulkanisation von Kautschuk 1851 stand ein Werkstoff zur Verfügung, der diese Anforderung erfüllte. Das Erscheinen des Kautschuk bewirkte nicht nur einen Fortschritt für die zahnärztliche Prothetik, sondern löste auch die Entwicklung der zahnärztlichen Kieferbruchschienung aus.

Der erste, der sich nach 1850 mit der prothetischen Versorgung eines Resektionspatienten auseinandersetzte, war der französische Zahnarzt Pierre Préterre ${ }^{10} .1858$ gab er seine Methode bekannt. Sie basierte auf der klinischen Tatsache, dass jede Kontinuitätstrennung des Unterkiefers von einer intermaxillären Occlusionsstörung begleitet war. Um diesen Zustand zu normalisieren, schuf Préterre im Unterkiefer einen künstlichen Zahnbogen, der die normale Verzahnung zum Oberkiefer wiederherstellte.

Die Préterrsche Methode kann nur im weitesten Sinne zur Resektionsprothetik gezählt werden, da sie weder eine Verbesserung der Ästhetik noch der Funktion brachte. Trotzdem war es das Verdienst Préterres, als erster auf die Problematik der prothetischen Versorgung nach Unterkieferresektionen hingewisen zu haben.

Nach Préterre waren es nicht Zahnärzte, die sich mit den funktionellen Auswirkungen der Resektionsoperationen befassten, sondern Chirurgen. Ihr Interesse galt den dislozierenden Kräften der Narbenschrumpfung. Um diesen entgegenzuwirken, klemmten sie Holz-, Elfenbein- oder Goldstäbchen zwischen die Kieferstümpfe ${ }^{11}$. Dadurch liess sich die Verschiebung weitgehend aufhalten, das funktionelle und aesthetische Problem bestand aber weiterhin. Erst als der französische Chirurg Léopold Ollier den Zahnarzt Claude Martin zur prothetischen Versorgung von Resektionspatienten heranzog, konnten diese Probleme teilweise gelöst werden ${ }^{12}$. Martin verwendete als Platzhalter nicht ein Holz- oder Goldstäbchen, sondern eine Immediatprothese aus Kautschuk. Diese setzte er 10 Tage nach der Operation ein. Infolge des direkten Kontaktes zwischen Prothese und Wundfläche traten aber Entzündungen und Sekretstauungen auf. Diese Komplikationen behob Martin, indem er auf der Unterseite der Prothese Drainagerillen einfräste. Nach der Abheilung des Operationsgebietes wurde die Immediatprothese durch eine definitive Kautschukprothese ersetzt. Anhand einer grossen Patientenzahl erkannte Martin, dass der Halt der Immediatprothesen - vor allem bei ausgedehnten Resektionen - schwer zu erreichen war. Für derartige Fälle schuf er die Immediatschiene (unmittelbarer Kieferersatz), deren Form dem resezierten Kieferstück entsprach. Diese Schiene befestigte Martin unmittelbar nach der Resektion mittels Drähten an den Kieferstümpfen. 
Die Schleimhaut wurde dabei nicht über der Schiene, sondern unterhalb dieser zusammengenäht, so dass ein Sekretabfluss gewährleistet war ${ }^{13}$. Bei Medialresektionen des Unterkiefers erhielt die Zunge an der Schiene einen neuen Halt. Als Gegenstück zur Immediatschiene entwickelten deutsche Zahnärzte wie Carl Sauer, Heinrich Boennecken oder Carl Partsch die Resektionsverbände ${ }^{14}$. Der Unterschied zur Immediatschiene zeigte sich vor allem in der Formgebung. Der Resektionsverband hatte die Gestalt eines Metallbügels, an dessen Enden zwei Halterungen zur Aufnahme der Kieferstümpfe angebracht waren.

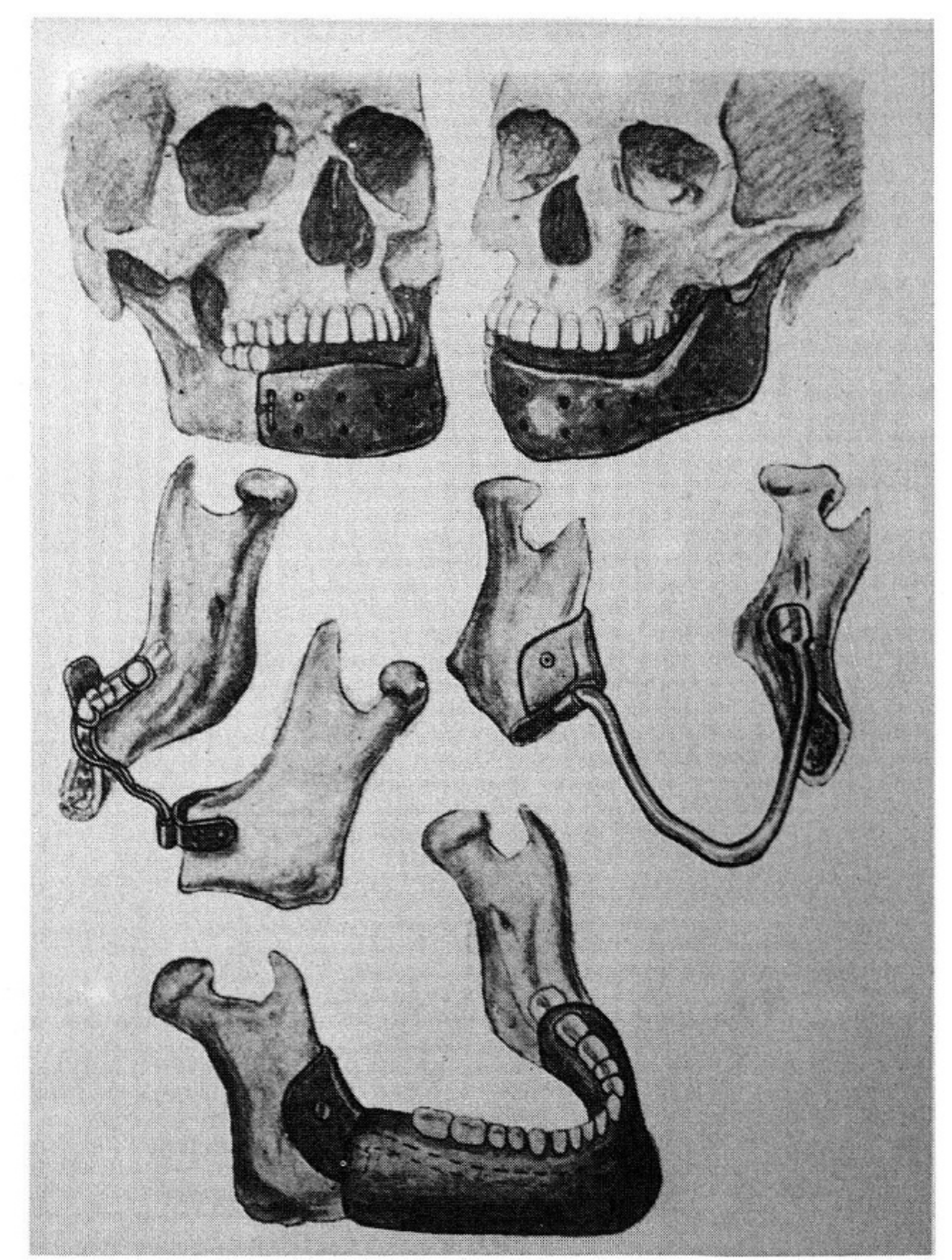

Abb.1: Unmittelbarer Kieferersatz: Im oberen Teil der Abb. Immediatschiene. Im mittleren Teil: Resektionsverband. Im unteren Teil: Resektionsverband mit Resektionsprothese. 
Nach der Abheilung des Operationsgebietes und der Erschöpfung des Narbenzuges wurden die Kieferschienen durch eine Resektionsprothese ersetzt.

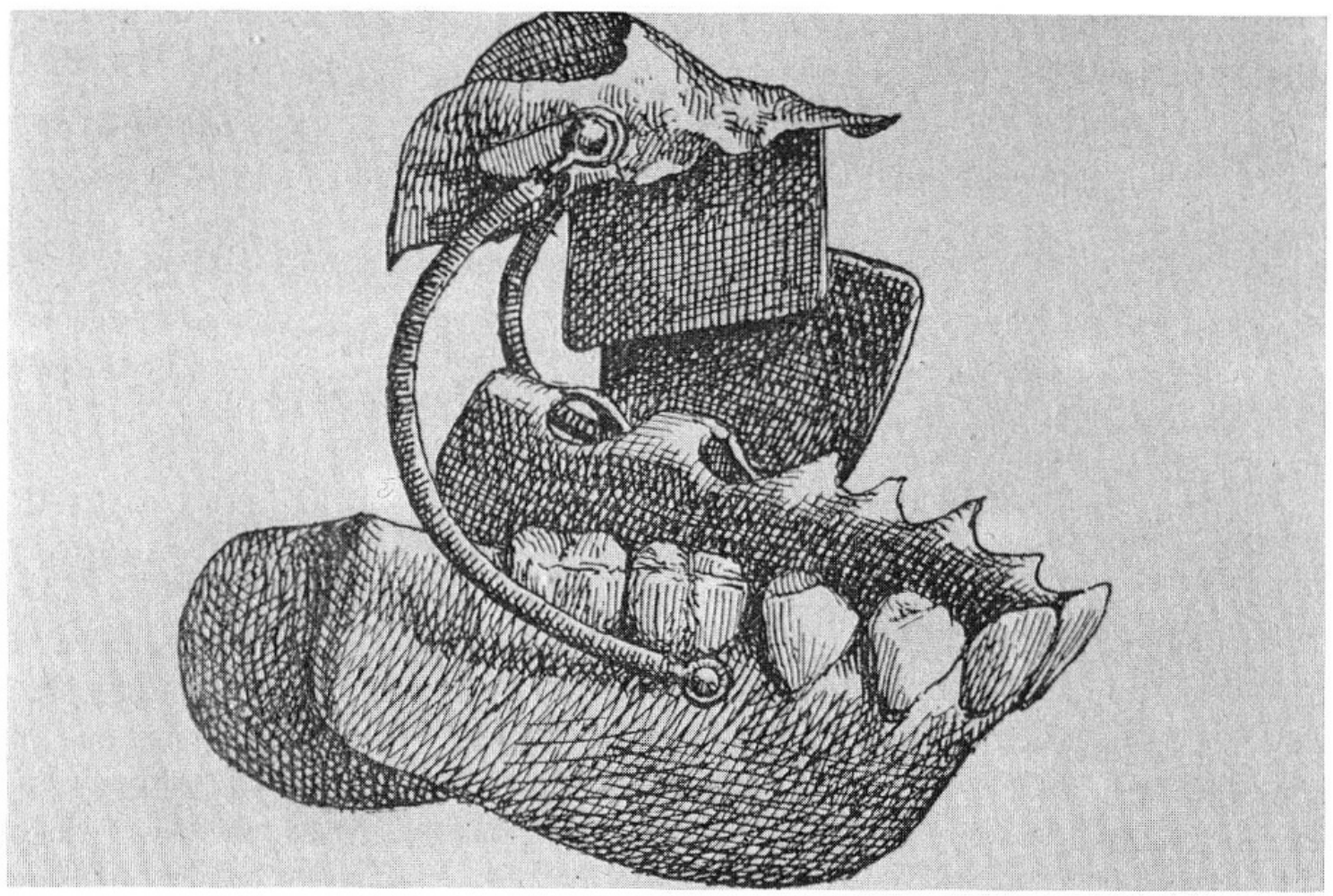

Abb.2: Unterkieferresektionsprothese. Um den Halt zu verbessern, wurde die Prothese durch Federn mit einer Gaumenplatte verbunden. Die Pelotten auf der linken Seite verhinderten ein Abdrängen der resezierten Unterkieferhälfte auf die gesunde Seite.

Der Resektionsverband hatte gegenüber der Immediatschiene den Vorteil, dass er aus Metall bestand und wie eine Barrenbrücke einige Millimeter über der Schleimhaut zu liegen kam. Die Kenntnis vom hygienischen Vorteil einer Metallfläche gegenüber dem Kautschuk veranlasste den Zürcher Professor Ambrogio Stoppany, eine Immediatschiene aus vergoldetem Metall herzustellen ${ }^{15}$.

Das Gegenteil zum unmittelbaren Kieferersatz bildete die orthopädische Methode ${ }^{16}$. Zwei Faktoren bestimmten ihre Entwicklung:

1. Die postoperativen Komplikationen, die die Immediatschiene und der Resektionsverband mit sich zogen.

2. Die apparativen Fortschritte der Kieferorthopädie.

Die orthopädische Behandlung setzte erst nach der Abheilung des Opera- 
tionsgebietes ein. Mit Hilfe von Schrauben und Federn wurden die Narben so lange gedehnt, bis sich die dislozierten Kieferstümpfe in die ursprüngliche Lage zurück reponieren liessen. Nach der Entfernung der kieferorthopädischen Apparaturen erhielt der Patient als Platzhalter eine Resektionsprothese. Das orthopädische Vorgehen war nur bei bezahnten Kieferstümpfen durchführbar.

Der unmittelbare Kieferersatz und die orthopädische Methode erzielten durch die nachträglich eingesetzte Resektionsprothese eine Verminderung der physiognomischen Entstellung.

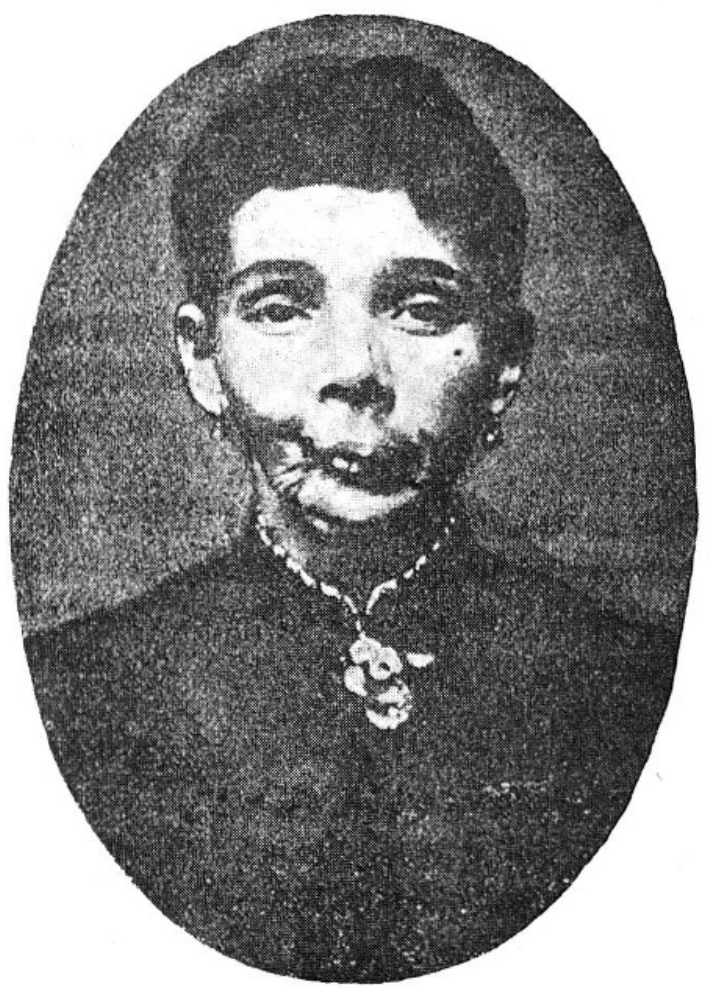

Abb.3: Patientin nach Teilresektion des rechten Unterkiefers.

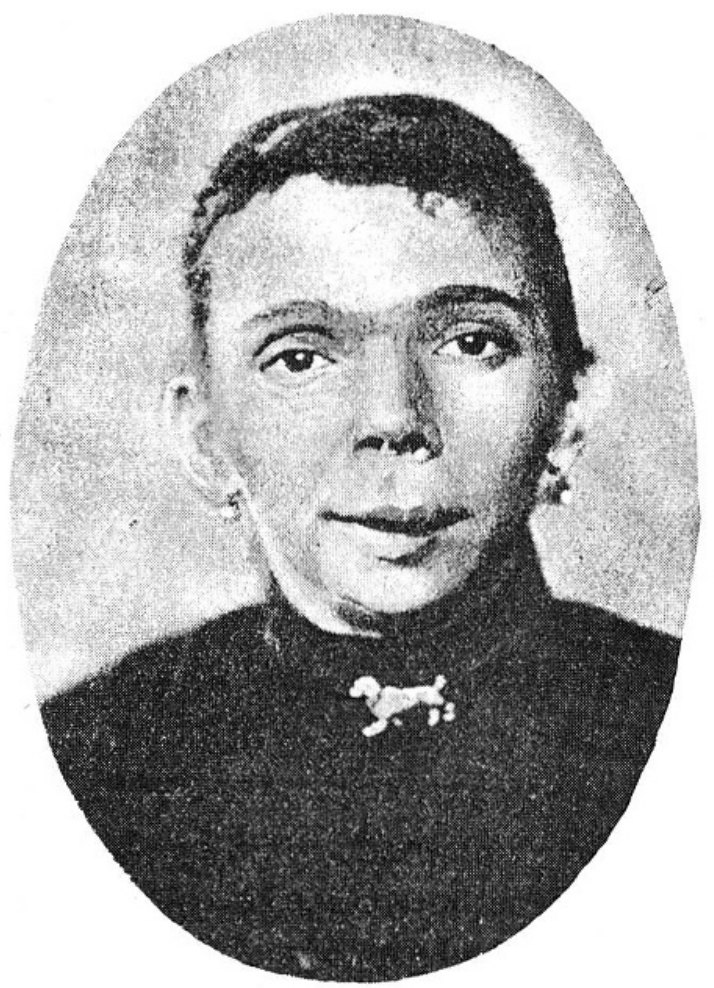

Abb.4: Gleiche Patientin wie Abb. 3 mit eingegliederter Resektionsprothese.

Problematisch blieb aber die Funktion der Prothese. Um sie zu verbessern, überbrückte 1895 der Berliner Chirurg Themistocles Gluck den Defekt mit einem Implantationssteg aus Gold, gestaltet nach dem Vorbild des Resektionsverbandes ${ }^{17}$. Neben Gold kam später auch Elfenbein zum Einsatz. In der Mehrzahl der Implantationsfälle kam es aber zu Knochenresorptionen 
oder Sequesterbildungen, so dass diese Methode vorerst wieder aufgegeben wurde.

In dem Versuch von Gluck, einen Resektionsverband zu implantieren, widerspiegelt sich die funktionelle Problematik der Resektionsprothetik im Unterkieferbereich. Durch eine dauerhafte und stabile Überbrückung des Resektionsdefektes wollte Gluck eine Optimierung des Prothesenlagers und damit eine verbesserte Funktion der definitiven Prothese erreichen.

\section{Die Erforschung der Vorgänge bei den Knochentransplantationen und ihre Auswirkungen auf die Knochenplastik am Unterkiefer}

Parallel zur Entwicklung der zahnärztlichen Resektionsprothetik setzten in der Chirurgie zielgerichtete Forschungen zur Klärung der Vorgänge bei Knochentransplantationen an den Extremitäten ein. Einer, der sich seit 1860 mit diesem Problem klinisch auseinandersetzte, war der französische Chirurg Léopold Ollier. 1891 gab Ollier - nach dreissigjähriger Forschungsarbeit - seine Resultate am Internationalen Medizinerkongress in Berlin bekannt. Seine Erkenntnisse lassen sich in zwei wichtige Punkte zusammenfassen ${ }^{18}$ :

1. Es besteht ein grosser Unterschied zwischen der Auto-, Homo- und Heteroplastik. Nur der körpereigene Knochen zeigt nach der Transplantation ein Überleben und Weiterwachsen. Die wichtigste Rolle bei der Transplantation spielt das Periost. Es bleibt auch nach der Verpflanzung lebens- und produktionsfähig. Jedes periostlose Transplantat verfällt der Resorption oder wird durch Bindegewebe abgekapselt.

2. Ein Erfolg bei der Transplantation ist nur mit artgleichem, lebendem und periostbedecktem Knochen gegeben.

Nach der Bekanntgabe dieser Resultate begann der Marburger Chirurg Arthur Barth die Ergebnisse Olliers mikroskopisch zu überprüfen. Barth ging es dabei vor allem um die Klärung der Frage: Bleibt das ganze Transplantat am Leben oder sterben Teile davon ab ? Seine Untersuchungen zeigten, dass nach der Transplantation das Periost und das Knochenmark abstarben. Wurde hingegen ein Transplantat in ein ossifikationsfähiges Lager eingesetzt, so wuchsen nach dem Tod des Transplantates Blutgefässe ein. Aufgrund dieser Revaskularisierung setzte die Bildung von neuem Knochengewebe ein, wobei das abgestorbene Transplantat als Gerüst und Kalklieferant diente ${ }^{19}$. Anhand dieser Erkenntnisse zog Barth den Schluss, 
dass es bei Vorliegen eines ossifikationsfähigen Lagers gleichgültig wäre, welches Transplantatmaterial verwendet würde.

Die gegensätzlichen Resultate von Ollier und Barth übten eine starke Hemmung auf die plastische Chirurgie aus ${ }^{20}$. Somit blieb die endgültige Klärung der Transplantationsvorgänge weiterhin offen. Diese Tatsache wirkte sich direkt auf die chirurgische Wahl der Transplantationstechnik am Unterkiefer aus. Als der Kölner Chirurg Bernhard Bardenheuer 1891 die erste Knochenplastik am Unterkiefer vornahm, zog er die StiellappenTechnik der freien Transplantation vor ${ }^{21}$. Bei seinem Vorgehen konnte Bardenheuer an die klinischen Erfahrungen der gestielten Hautlappentechnik anknüpfen. Bardenheuer bildete einen vor dem Ohr gestielten Haut-PeriostKnochenlappen. Das freie Ende dieses Lappens verlagerte er von der Stirn in die Mundhöhle, wobei die Epidermisfläche des Lappens der Mundschleimhaut zugekehrt wurde. Das Periost des Stirnlappens vernähte er mit dem Periost der Kieferstümpfe. Bardenheuers Vorgehen war erfolgreich. Es kam zu einer festen Verbindung zwischen dem Transplantat und den Kieferstümpfen. Bardenheuers Technik hatte aber den Nachteil, dass der Patient im Bereich des Os frontale einen bleibenden Defekt hinnehmen musste. Wegen dieser Entstellung gab Bardenheuer die Stirnlappenbildung auf ${ }^{22}$. In zwei Fällen verwendete er einen gestielten Haut-Periost-Knochenlappen aus der gesunden Seite des Unterkiefers.

Die guten Resultate von Bardenheuer bewirkten, dass auch andere Chirurgen die gestielte Knochenplastik anwandten. Zwischen 1892 und 1900 wurde diese Technik zehnmal in Europa ausgeführt ${ }^{23}$. Dabei waren sechs Erfolge zu verzeichnen. In keinem der zehn Transplantationsfälle wurde eine Kieferschienung vorgenommen.

Im Jahre 1900 nahm der Moskauer Chirurg Sykoff, unbeachtet der noch bestehenden Kontroverse zwischen Ollier und Barth, die erste freie Knochenplastik am Unterkiefer vor ${ }^{24}$. Das periostbedeckte Transplantat, das Sykoff der gesunden Seite des Unterkiefers entnommen hatte, heilte komplikationslos ein. Sykoffs Erfolg rief aber in der chirurgischen Fachwelt keine Reaktion hervor. Erst sieben Jahre später nahm Erich Lexer das Vorgehen von Sykoff wieder auf. Zwischen 1907 und 1908 führte Lexer - mit Erfolg - 3 freie Knochentransplantationen am Unterkiefer durch ${ }^{25}$. Als Transplantate verwendete er periostbedeckte Tibia-, Rippen- und Unterkieferknochen. Lexer war der erste Chirurg, der die Notwendigkeit einer präoperativen Kieferschienung erkannte.

Parallel zu Lexers Knochenplastiken fällt auch die Klärung der gegen- 
sätzlichen Ansichten von Ollier und Barth. Dies war das Verdienst des Berliner Chirurgen Georg Axhausen. Er konnte beweisen, dass beim Absterben eines autologen Transplantates Teile des Periostes und des Knochenmarks am Leben blieben. Deren osteogenetische Fähigkeiten sorgten für die Knochenneubildung im Transplantat. Daraus folgerte Axhausen, dass der körpereigene, lebende periostbedeckte Knochen das ideale Material zur Verpflanzung sei ${ }^{26}$. Aufgrund dieser Erkenntnis verwarf Barth 1908 seine Hypothese, das ganze Transplantat sterbe ab, und schloss sich der Ansicht Axhausens an ${ }^{27}$.

Zwischen 1908 und 1914 wurden in Europa elf freie Knochenplastiken am Unterkiefer durchgeführt. Als Transplantate wurden in sechs Fällen Rippenknochen, in vier Fällen Tibiaknochen und in einem Fall Unterkieferknochen verwendet. Von diesen elf Transplantaten heilten nur drei fest ein ${ }^{28}$. Im Gegensatz zur Knochenplastik an den Extremitäten nahmen die Chirurgen - ausser Erich Lexer - keine Ruhigstellung der Kieferstümpfe vor ${ }^{29}$.

\section{Der Erste Weltkrieg und der Durchbruch der freien Knochenplastik}

Kiefer-Gesichtsverletzungen hatten in den Kriegen vor dem Ersten Weltkrieg eine geringe kriegschirurgische Bedeutung, da sie nur 0,5-1 Prozent der Gesamtverletzungen ausmachten ${ }^{30}$. Deshalb schenkten die Armeen Europas dem Aufbau einer kriegszahnärztlichen Versorgung zu wenig Beachtung ${ }^{31,32}$. Dies wirkte sich in den ersten Monaten des Weltkrieges schwerwiegend aus. Die neue Waffentechnik sowie die moderne Körperdeckung, bei welcher der Wehrmann nur noch seinen Kopf als Schussfeld freigab, liessen die Zahl der Kiefer-Gesichtsverletzungen und das Ausmass der Verwundungen stark ansteigen ${ }^{33}$. Die neue Kriegstaktik veranlasste die kriegsführenden Länder, sofortige sanitätsdienstliche Massnahmen zu treffen. Es entstanden spezialisierte Einrichtungen für die Behandlung von Kiefer-Gesichtsverletzten : die Kieferschussstationen, die den Reservelazaretten angegliedert waren ${ }^{34}$.

Als Leiter einer Kieferschussstation bestimmte die deutsche Heeresleitung jeweils einen Zahnarzt, dem einige Zahnärzte und Zahntechniker unterstellt waren. Für spezielle chirurgische Eingriffe standen dem Leiter die Chirurgen und Augenärzte der Reservelazarette zur Verfügung ${ }^{35}$.

Die Erfahrungen aus den ersten Kriegsmonaten zeigten, dass die Wiederherstellung von Kiefer-Gesichtsverletzten nur durch einen systematischen 
Behandlungsablauf erreicht werden konnte. Dieser gliederte sich in sechs Phasen ${ }^{36}$ :

1.Phase: Notfalleingriffe, psychische Betreuung.

2. Phase: Erstellen der Krankengeschichte.

3. Phase: Wundbehandlung.

4. Phase: Kiefer- und Defektbruchbehandlung.

5. Phase: Wartezeit.

6. Phase: Knochen- und Weichteilplastik.

Zu Beginn des Krieges wandten die Chirurgen zur Primärversorgung von Unterkieferdefektbrüchen die Methoden aus der Friedenszeit an : die Immediatschiene und den Resektionsverband. In den meisten Fällen kam es aber zu Eiterungen und Sequesterbildungen, so dass die Schienen wieder entfernt werden mussten. Diese Erfahrungen liessen die kriegschirurgische Indikation für Immediat- oder Resektionsschienen auf glatt durchtrennte Fragmente einschränken ${ }^{37}$. Für die Primärversorgung von Defektbrüchen entwickelten die Zahnärzte sogenannte Stützprothesen ${ }^{38}$. Ihre Befestigung erfolgte nicht mehr an den Kieferstümpfen, sondern an der Restbezahnung.

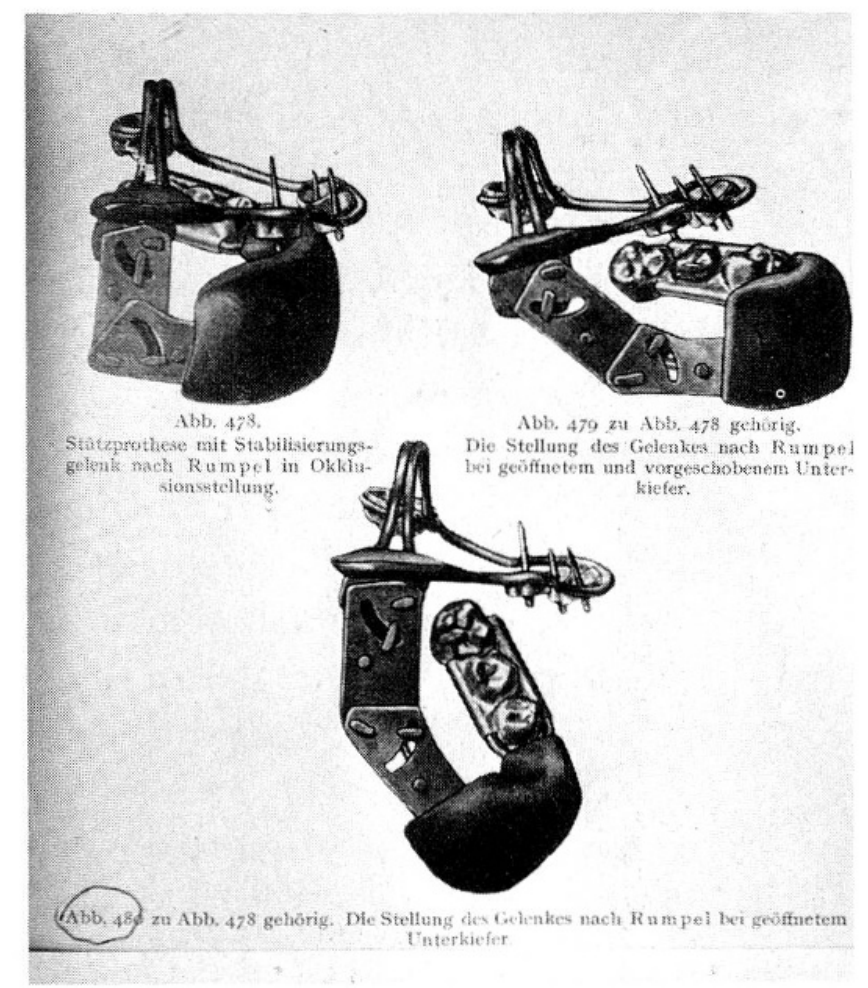

Abb.5 : Stützprothese mit Gelenk zur primären Versorgung eines einseitigen Unterkiefer-Gelenkabschlusses. Dieser Apparat wurde einerseits an der vorhandenen Unterkiefer-Restbezahnung befestigt, andererseits mittels Wurzelstiftkappen am Oberkiefer. 


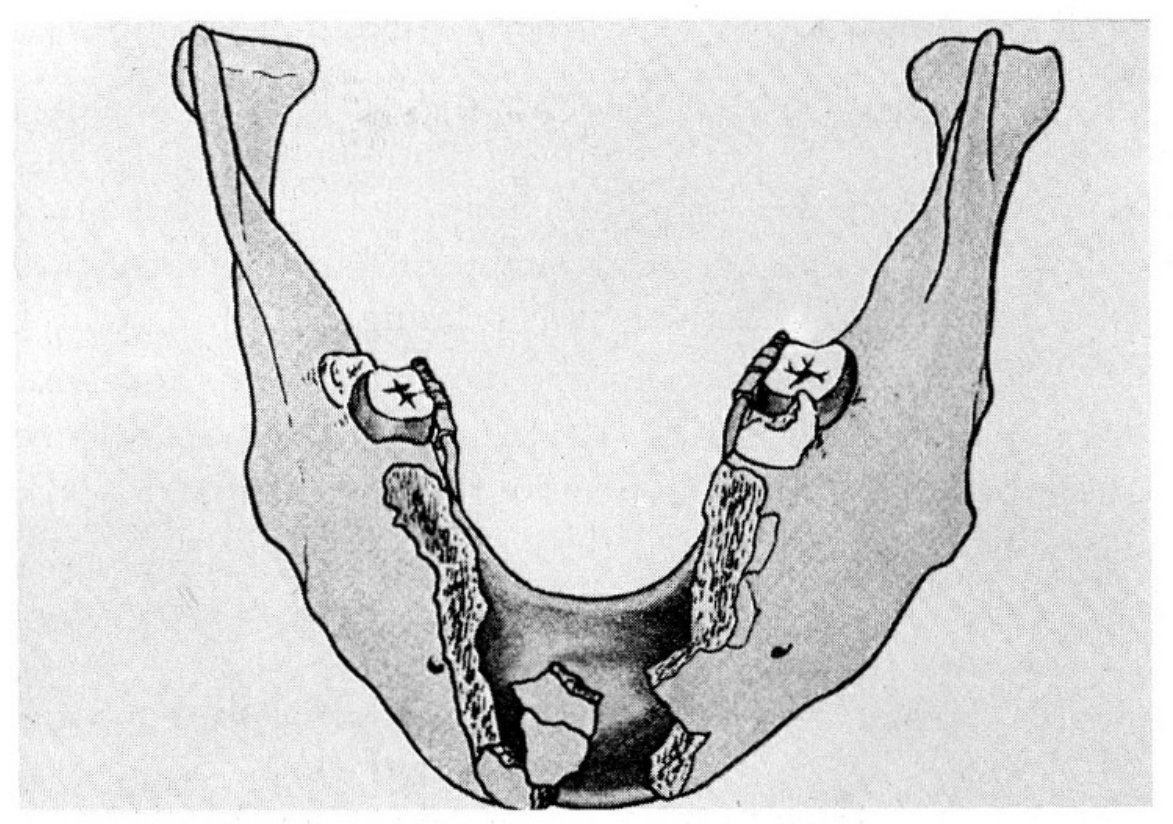

Abb.6: Stützprothese zur primären Versorgung einer medialen Unterkieferdefektfraktur. Befestigung mittels Molarenbänder.

Beim zahnlosen Unterkiefer erhielt die Prothese - entfernt von der Verletzungstelle - an den Kieferstümpfen ihren Halt, oder es kam die extraorale Nagelung zur Anwendung ${ }^{39}$.

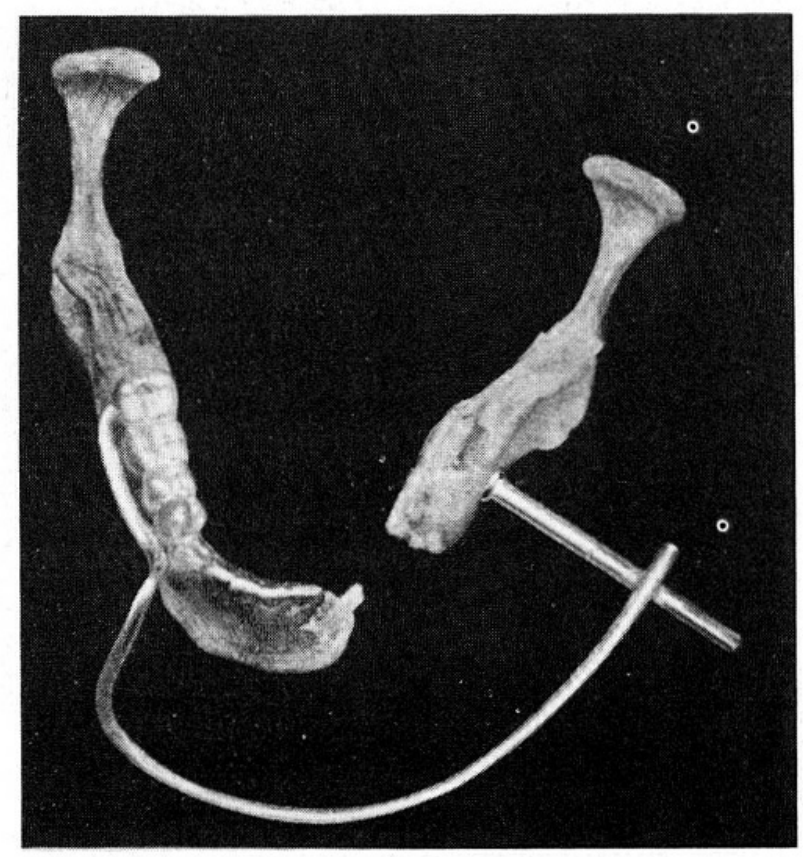

Abb.7 : Fixationsverband im zahnlosen Unterkiefer : extraorale Nagelung. 
In ihrer Funktion unterschied sich die Stützprothese nicht von der Immediatschiene. Beide dienten der Knochen- und Weichteilstützung. Wie die Immediatschiene erforderte auch die Stützprothese eine intensive Hygiene und Nachkontrolle. Nach der Abheilung der verletzten Gewebe setzte nicht sofort die definitive Rekonstruktion ein, sondern das abgeheilte Gebiet wurde noch während einiger Wochen (ca. 8-12 Wochen) beobachtet. Traten keine Spätkomplikationen auf, so konnte die definitive Rekonstruktion der Defekte vorgenommen werden ${ }^{40}$. Bei ausgedehnten Knochenverlusten diente die Stützprothese als Vorlage für die Rekonstruktion des Knochengerüstes und der Weichteile.

Der grosse Anfall an Verletzten mit Unterkiefer-Knochenverlusten schuf unerwartete Voraussetzungen zur Einarbeitung in die Technik der freien Knochenplastik. Dabei hatten die deutschen Chirurgen den Vorteil, dass ihnen diese Thematik schon etwas vertraut war; denn in der Vorkriegszeit war die deutsche Plastische Chirurgie - europäisch betrachtet - führend in der klinischen Bearbeitung der Knochenplastik an den Extremitäten und am Unterkiefer. Die Chirurgen der Kieferlazarette knüpften an jenen Erfah-

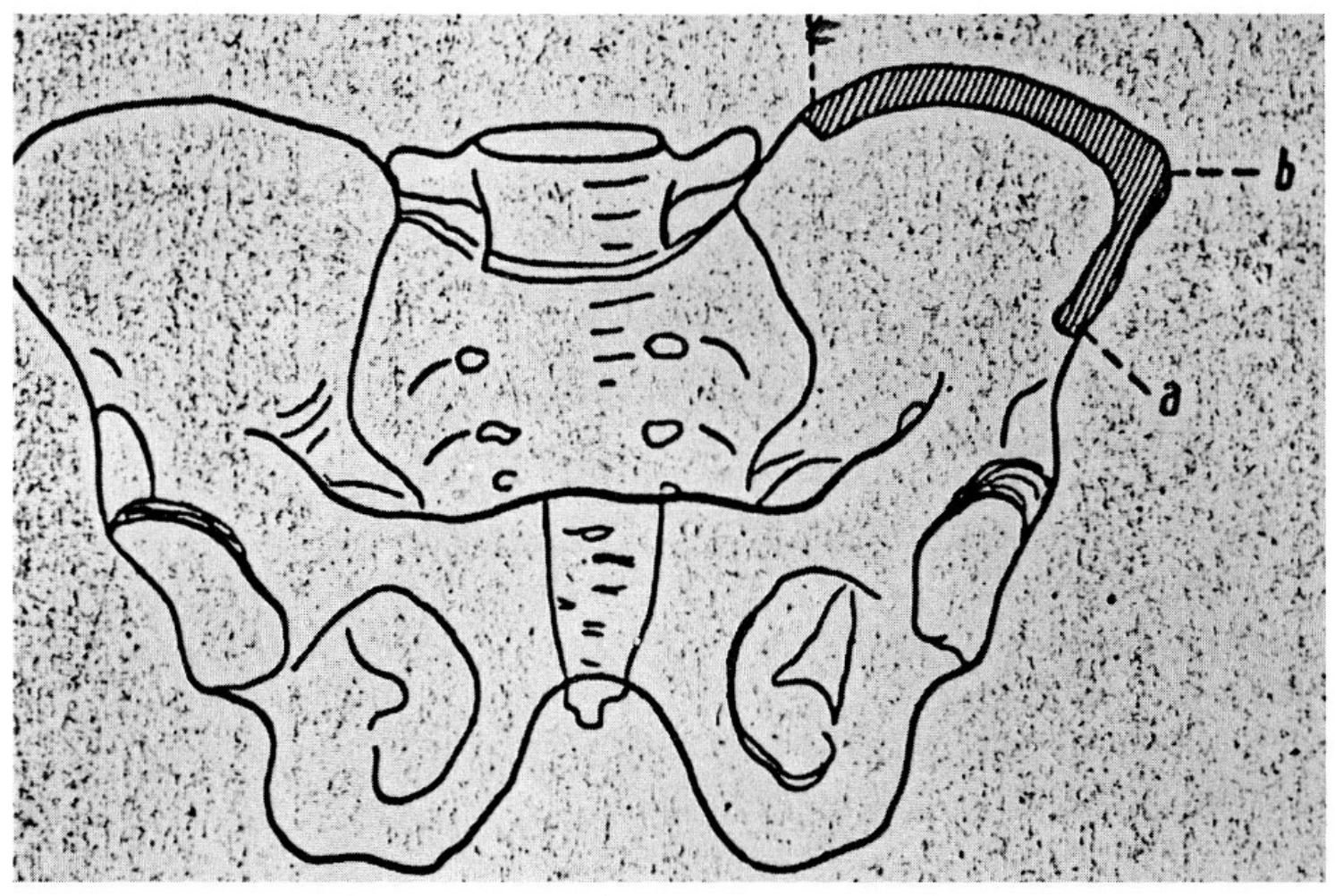

Abb.8: Beckenkamm zur Rekonstruktion einer Unterkieferhälfte : a ergibt Gelenkende, b Kieferwinkel, $c$ Kinnende. 
rungen an und konnten sie durch die Anwendung an einem grossen Patientenmaterial erweitern.

Als Transplantate wurde das bereits bekannte Knochenmaterial der Tibia und der Rippe verwendet. Neu hinzu kam der Beckenkamm. Seine Auswahl ist dabei keine kriegschirurgische Innovation, sondern beruht auf einer - 1911 geäusserten - Empfehlung des Kieler Chirurgen Rudolf Goebell ${ }^{41}$.

Ab 1916 empfahlen die Chirurgen August Lindemann und Rudolf Klapp für die Rekonstruktion eines Unterkieferteildefektes oder einer Unterkieferhälfte nur noch Beckenkamm ${ }^{42}$. Als Vorteile gaben sie an : Einfacher Zugang zur Entnahmestelle, die Möglichkeit einer leichten Formgebung und der grosse Markgehalt des Beckenkammes ${ }^{43}$.

Für die Rekonstruktion des aufsteigenden Astes und des Gelenkkopfes schlug Rudolf Klapp 1916 den IV. Mittelfussknochen vor ${ }^{44}$.

Die Auswertung der kriegschirurgischen Literatur zeigt, dass die Chirurgen bei der Durchführung der ersten freien Transplantationen das Schwergewicht auf die Verankerung des Transplantates am Kieferknochen legten.

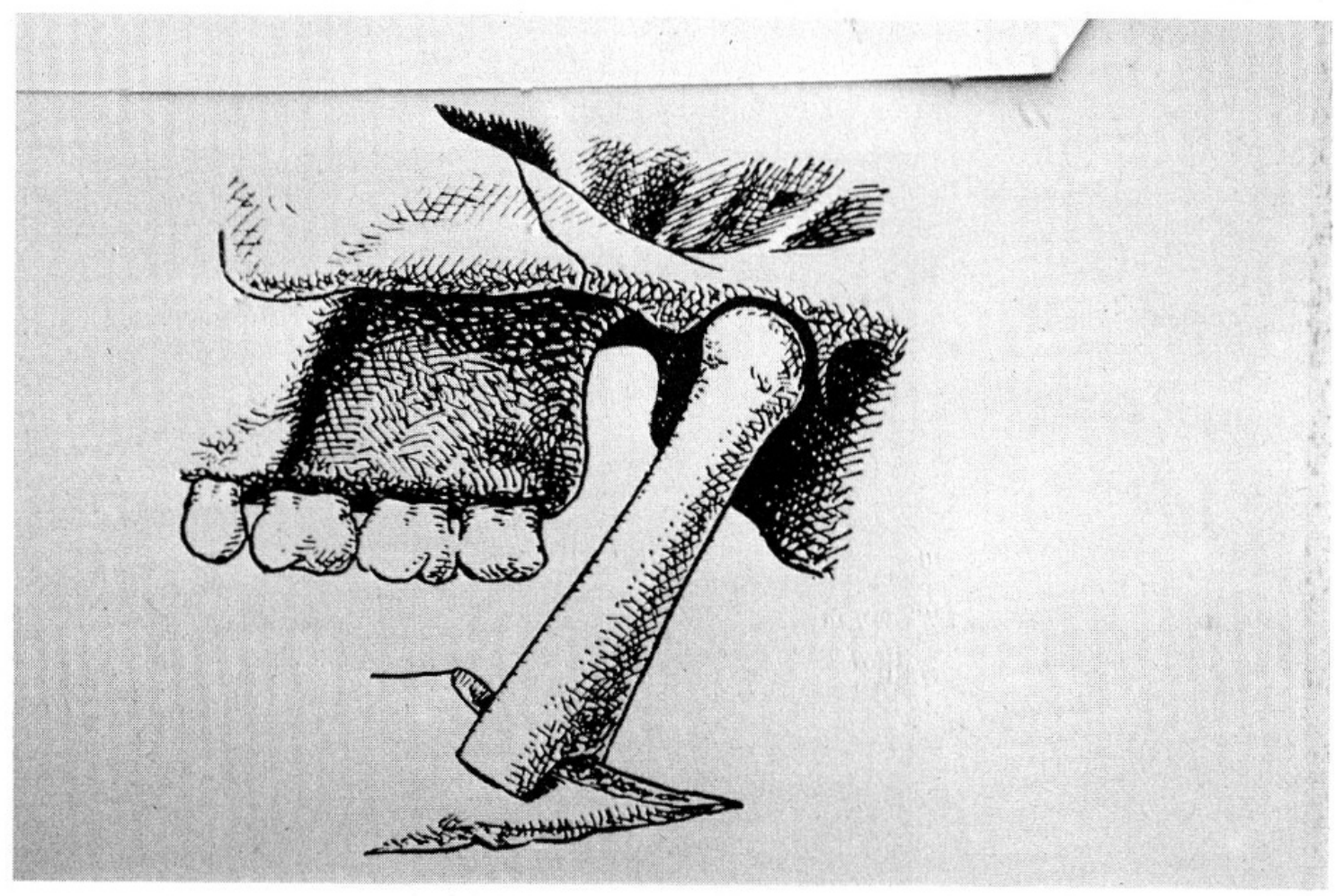

Abb.9: Metatarsus IV in situ: Befestigung am Unterkieferstumpf durch Einkeilung. 
Daraus resultierten verschiedene Befestigungmethoden, wie z. B. die Drahtnaht, die Einkeilung, das Goldband und die Einbolzung. Bei dieser Technik wurden die Transplantatenden zuerst dornenförmig zugeschnitten und danach in die vorbereiteten Markhöhlungen der Kieferstümpfe eingeschoben $^{45}$.

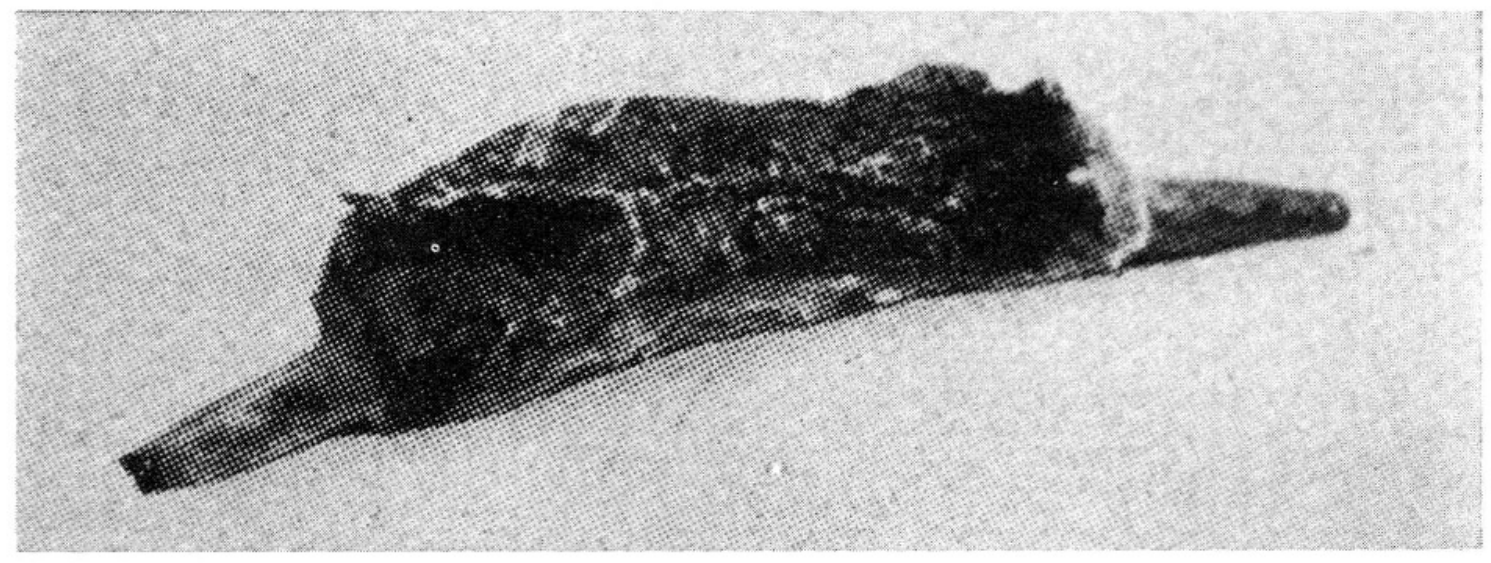

Abb.10: Befestigung des Transplantates durch Einbolzung.

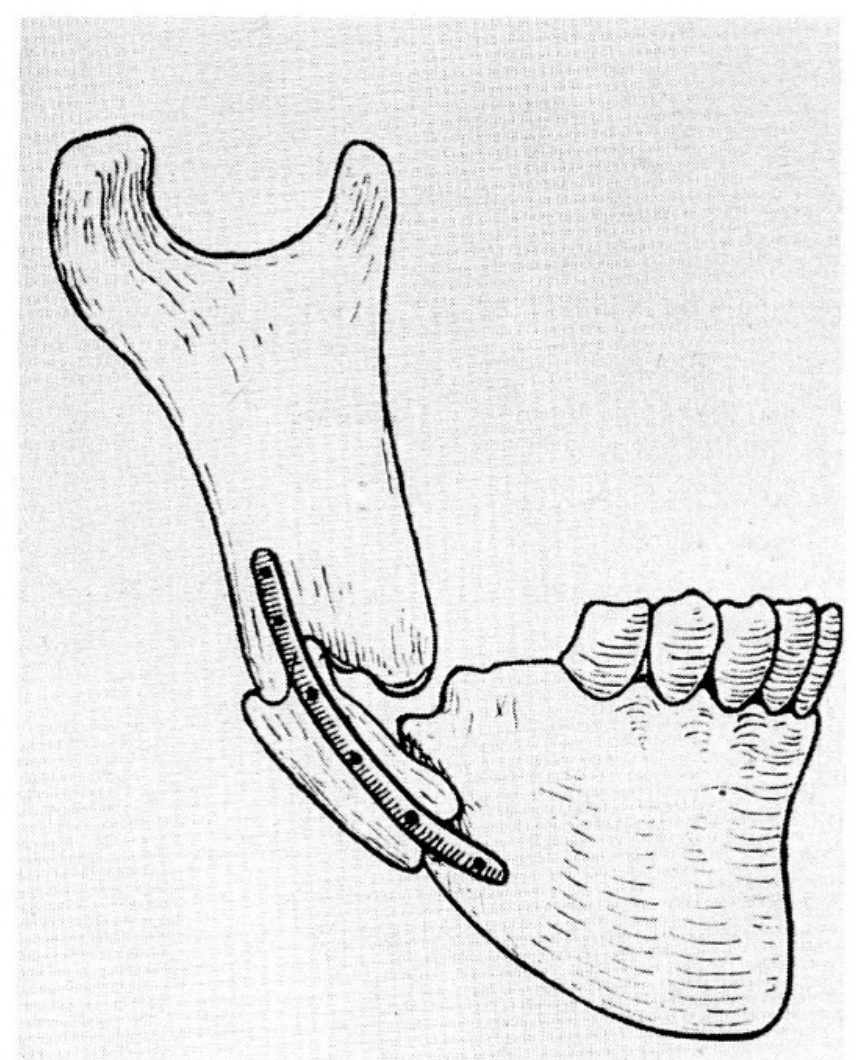

Abb.11: Befestigung des Transplantates durch Goldband. 
Schon in den ersten Kriegsmonaten zeichnete sich eine grundlegende Veränderung im chirurgischen Transplantationskonzept ab. Ausschlaggebend dafür war die vom Kriegsgeschehen erzwungene Zusammenarbeit zwischen Chirurgen und Zahnärzten, eine Tatsache, die im 19. Jahrhundert oder zu Beginn des 20. Jahrhunderts nur in vereinzelten Fällen zustande gekommen war $^{46}$. Diese Zusammenarbeit brachte den Chirurgen die Erkenntnis, dass eine komplikationslose Kieferbruchbehandlung eine stabile Kieferschienung und eine klare Prognose für die dem Bruchspalt benachbarten Zähne erforderte ${ }^{47}$; zwei Faktoren, die bis anhin nicht nur in der chirurgischen Kieferbruchtherapie, sondern auch bei der Durchführung der Knochentransplantationen unbeachtet geblieben waren. Bis Ende 1915 hatten sich diese Voraussetzungen bei den Chirurgen eingebürgert. Hingegen herrschte bis zum Kriegsende Unklarheit über die Ausdehnung der Kieferschienung. Die einen Chirurgen liessen nur den Unterkiefer schienen, andere verlangten - bei ausgedehnten Knochenverlusten - zusätzlich eine intermaxilläre Fixation für 6-8 Wochen ${ }^{48}$. In den Fällen, in denen die Stützprothese eine stabile, rotationsfreie Verbindung der Kieferstümpfe herstellen konnte, übernahm diese Prothese die Funktion der Kieferschienung. Anhand der klinischen und röntgenologischen Nachkontrollen der operierten Verletzten bildete sich die Regel heraus, dass die Unterkieferschienung nach 6 Monaten entfernt werden konnte. War es in diesem Zeitraum nicht zu einer festen Einheilung des Transplantates gekommen, so versuchte man die Knochenbildung durch Beklopfen oder Fibrininjektionen anzuregen ${ }^{49}$. August Lindemann, Chirurg im Düsseldorfer Kieferlazarett, hatte zwischen 1915 und 1917282 freie Transplantationen vorgenommen. Darunter konnte er 152 Erfolge, 72 Teilerfolge und 58 Misserfolge verzeichnen ${ }^{50}$.

Die Transplantationsoperation wurde strikte von extraoral ausgeführt. Dabei galt der Grundsatz, dass die Mundhöhle nie eröffnet werden dürfe. Kam es während der Operation zu einer oralen Perforation, so wurde der Eingriff sofort abgebrochen und auf einen späteren Zeitpunkt verschoben ${ }^{51}$. Die operative Entnahme des Knochenstückes wie auch sein Einfügen ins Transplantatbett erfolgten stets in Lokalanästhesie (Novocain-Suprarenin), wobei der Patient präoperativ mit Morphium und Skopolamin sediert wurde $^{52}$.

Bis zum Kriegsende waren sich die Chirurgen nicht einig über das operative Rekonstruktionsvorgehen. Es gab Chirurgen, die zuerst die Weichteilkontinuität über den Stützprothesen wiederherstellten und erst sekundär das skelettale Gerüst ${ }^{53}$. Andre Chirurgen wie z.B. Lindemann 
gingen gerade umgekehrt vor. Das Vorgehen Lindemanns wurde nach dem Krieg zur allgemeinen gültigen Regel in der sich entwickelnden europäischen Kieferchirurgie.

\section{Schluss}

Die Resektionsprothetik bildete bis zum Ersten Weltkrieg die sicherste Massnahme zur Versorgung ausgedehnter Knochenverluste am Unterkiefer. Parallel zu ihrer Entwicklung wurden in der Chirurgie die ersten Knochenplastiken an den Extremitäten vorgenommen. Daraus ergaben sich auch Voraussetzungen für die skelettale Rekonstruktion kleiner Unterkieferdefekte. Da den Chirurgen aber ein zahnärztliches Verständnis fehlte, bestand das Transplantationskonzept nur aus der Fixation des Transplantates an den Kieferstümpfen. Erst die Zusammenarbeit zwischen Chirurgen und Zahnärzten im Ersten Weltkrieg brachte den entscheidenden Fortschritt für die skelettale Wiederherstellung eines Unterkieferdefektes. Dazu zählte nicht nur die Weiterentwicklung des unmittelbaren Kieferersatzes zur Stützprothese, sondern auch die Vervollständigung des bestehenden Transplantationskonzeptes durch die Ruhigstellung der Kieferstümpfe. Das zunehmende Verständnis für die gegenseitige Abhängigkeit von Chirurgie und Zahnheilkunde bei der Wiederherstellung von Kieferverletzten löste nach dem Ersten Weltkrieg die Entwicklung der europäischen Kieferchirurgie aus. 


\section{Anmerkungen}

1 Dupuytren, G., Leçons orales de clinique chirurgicale, Bd. 4. Paris 1834, S.626-688.

2 Hoffmann-Axthelm, W., Die Unterkieferresektion des Barons Dupuytren anno 1812. War sie die erste? In: Deutsche Zschr. für Mund-, Kiefer- und Gesichtschirurgie 12 (1988), 431. Hoffmann-Axthelm weist darauf hin, dass schon im 18. Jahrhundert eine Unterkieferresektion vorgenommen wurde. Dabei spielt die Frage, welche Resektion die erste gewesen sei, keine Rolle, denn die Resektion von Dupuytren löste die Entwicklung planmässiger Resektionen aus.

3 Perthes, G., Brochers, Ed., Verletzungen und Krankheiten der Kiefer. In: Neue Deutsche Chirurgie, Bd. 53. Stuttgart 1932, S.551.

4 Ibid. Signorini nahm die Totalexstirpation wegen einer Phosphornekrose des Unterkiefers vor.

5 Pitha, F., Billroth, Th., Handbuch der allgemeinen und speziellen Chirurgie, Bd.3., Stuttgart 1866, S. $311 \mathrm{f}$.

6 Dieffenbach, J. F., Die operative Chirurgie. Leipzig 1848, S. 53, 55 f.

7 Emmert, C., Lehrbuch der Chirurgie. Stuttgart 1860, S. 278.

8 Hoffmann-Axthelm, W., Die geschichtliche Entwicklung der Mund-, Kiefer- und Gesichtschirurgie. In: Fortschritte der Kiefer-Gesichtschirurgie, Bd. XXI. Stuttgart 1976, S.1-8. Hoffmann-Axthelm, W., Zur geschichtlichen Entwicklung des Knochenersatzes im Unterkiefer. In: Aufklärung, Fehler und Gefahren in der Mund-Kiefer-Gesichts-Chirurgie, Bd. XXX. Stuttgart 1985, S. 168-171.

Hoffmann-Axthelm, W., The Treatment of Maxillofacial Fractures and Dislocations in Historical Perspective. In: Krüger, E., Schilli, W., Oral and Maxillofacial Traumatology. Chicago 1982, S.38-40.

9 Schmocker, R., Die Unterkieferrekonstruktion. Ein geschichtlicher Überblick. In: Swiss Dent 10 (1989), 23-31. Schmocker argumentiert in der Einteilung seiner Arbeit fragwürdig, indem er festhält, dass die Kriterien zur Unterkieferrekonstruktion aus dem 1. Weltkrieg nicht den heute gültigen Auffassungen entsprächen. Ohne die wichtige Kriegsliteratur zu konsultieren, zitiert Schmocker die falsche Feststellung, dass es im 1. Weltkrieg nur selten einen klinischen Erfolg gegeben habe. Der Arbeit von Schmocker fehlt jegliche historische Relevanz.

10 Stoppany, G. A., Beitrag zur Prothesentherapie dislozierter Kieferfragmente nach Kontinuitätsresektion der Mandibula. In: Schweiz. Vierteljahresschrift für Zahnheilkunde 8 (1898), $14 \mathrm{f}$.

11 op. cit. sub 5, S. 312 .

12 Hoffmann-Axthelm, W., Geschichte der Zahnheilkunde (2. Auflage). Berlin 1985, S. 395.

13 Martin, C., Des résultats éloignés de la prothèse immédiate dans les résections du maxillaire inférieur. Lyon 1893.

14 Stoppany, G.A., Beitrag zum unmittelbaren Kieferersatz nach Continuitätsresektionen der Mandibula. In: Deutsche Mschr. für Zahnheilkunde 10 (1900), 54-57.

15 Ibid, S.59, 65.

16 Op. cit. sub 10, S.23-28.

17 Perthes, G., Die Chirurgie der Zähne, des Zahnfleisches und der Kiefer. In: Handbuch der praktischen Chirurgie, Bd.1. Stuttgart 1913, S.854. 
18 Ollier, L., De l'ostéogénèse chirurgicale. In: Verhandlungen des 10. Internationalen Medizinkongresses, Berlin 1891, S.2-31.

19 Barth, A., Über Osteoplastik in historischer Beziehung. In:'Langenbecks Arch. klin. Chir. 48 (1894), 466-477.

20 Lexer, E., Die Freien Transplantationen. In: Neue Deutsche Chirurgie, Bd. 26b, 2.Teil. Stuttgart 1924, S.11.

21 Bardenheuer, B., Über Unter- und Oberkieferresektionen. In: Verhandlungen Deutsche Gesellschaft für Chirurgie 21 (1892), 123-130.

22 Op. cit. sub 3, S. 563.

23 Klapp, R., Gesichts- und Kieferschüsse. In: Handbuch der ärztlichen Erfahrungen im Weltkrieg 1914/1918, Bd. 1. Leipzig 1922, S.519.

24 Lexer, E., Wiederherstellungschirurgie. Leipzig 1919, S. 77.

25 Ibid, S. $78 \mathrm{f}$.

26 Axhausen, G., Histologische Untersuchungen über Knochentransplantationen am Menschen. In: Deutsche Zschr. Chir. 91, (1908), 388-430.

27 Op. cit. sub 20, S. 12.

28 Op. cit. sub 23.

29 Diese Feststellung ergibt sich aus der vorhandenen Literatur zur Knochenplastik am Unterkiefer vor 1914. Hoffmann-Axthelm gibt in seiner Arbeit «Zur geschichtlichen Entwicklung des Knochenersatzes im Unterkiefer» (s. Anm. 8) nur Erich Lexer an.

30 Schulz, C. D., Zur Geschichte der Militärzahnmedizin in Deutschland vor dem 1. Weltkrieg. In: Wehrmed. Mschr. 33 (1989), Heft 4, S. 183.

31 Rose, O., Die zahnärztliche Tätigkeit im Zukunftskriege. In: Deutsche Mschr. Zahnheilkunde 30 (1912), $737 \mathrm{f}$.

32 Op. cit. sub 29, Heft 1, S. 37-40, Heft 3, S. 136-138, Heft 4, S.176-183, Heft 5, S. 228-230.

33 Römer, O., Lickteig, A., Die Kriegsverletzungen der Kiefer. Strassburg i. E. 1917, S. 202 f.

34 Wegen dem grossen Anfall von Kiefer-Gesichtsverletzten wurden ab 1915 spezielle Kieferlazarette errichtet.

35 Ganzer, H., Bericht über die Tätigkeit der Kieferschussstation im Reservelazarett Hochschule für die bildenden Künste in Charlottenburg. In: Deutsche Mschr. Zahnheilkunde 33 (1915), S. 449f.

36 Ibid, S.450-454.

37 Op. cit. sub 29, S.230f.

38 Misch, J., Rumpel, C., Die Kriegsverletzungen der Kiefer und der angrenzenden Teile. Berlin 1916, S.420-427.

39 Op. cit. sub 29, S.254.

40 Op. cit. sub 36, S.458. Heute kann diese Wartezeit durch die Antibiotika stark verkürzt werden.

41 Göbell, R., Zum plastischen Ersatz von angeborenen und erworbenen Unterkieferdefekten. In: Deutsche Zschr. Chir. 123 (1913), S. 144-178.

42 Hoffmann-Axthelm, W., Zur geschichtlichen Entwicklung des Knochenersatzes im Unterkiefer. In: Aufklärung, Fehler und Gefahren in der Mund-Kiefer-Gesichts-Chirurgie, Bd. XXX. Stuttgart 1985, S. 169.

43 Op. cit. sub 3, S. 564.

44 Op. eit. sub 40, S. 169. 
45 Op. cit. sub 36, S.459-462.

46 Auf die Problematik der Arzt-Zahnarzt-Beziehung geht Schulz in seiner Arbeit «Die Geschichte der Militärzahnmedizin in Deutschland vor dem 1. Weltkrieg» (s. Anm.32) ein. Ebenfalls befassen sich damit Paff und Schoenbeck in ihrem Buch : Kursus der zahnärztlichen Kriegschirurgie und Röntgentechnik. Leipzig 1916, S. 15 f. Aus beiden Arbeiten wird ersichtlich, dass die Ärzteseite und das Kriegsministerium sich gegen einen Einsatz der Zahnärzte in der Armeesanität wehrten. Deshalb organisierten einige Zahnärzte selbst kriegszahnärztliche Kurse. Nach dem Ausbruch des 1. Weltkrieges wurden diese Kurse von der Armeesanität übernommen (s. Anm. 36, S. III, IV).

47 Kariöse Zähne wurden zuerst konservierend versorgt. Zähne, die eine röntgenologische Veränderung der periapikalen Region zeigten, wurden extrahiert.

48 Op. cit. sub 36, S. 468 f.

49 Ibid, S. 467.

50 Op. cit. sub 29, S. 264.

51 Diese Massnahme war nötig, um abzuklären, ob der Speichelzutritt eine Entzündung des Operationsgebietes auslöste. Heute wird die Transplantationsoperation - dank der antibiotischen Abschirmung - von intraoral durchgeführt. Dadurch können auch kosmetisch störende Operationsnarben vermieden werden.

52 Op. cit. sub 36, S.162 f., 458.

53 Schröder, H., Über wichtige und strittige Punkte der modernen Kieferbruchbehandlung. In: Deutsche Mschr. Zahnheilkunde 35 (1917), 483, 488-491.

\section{Abbildungsnachweise}

Abb. 1 Pfaff, H. W., Schoenbeck, F., Kursus der zahnärztlichen Kriegschirurgie und Röntgentechnik. Leipzig 1916, S.174, Fig. 73.

Abb.2 Martin, C., Des Résultats éloignés de la Prothèse immédiate. Paris 1893, S.62, Fig. 16.

Abb. 3 Ibid, S. 80, Fig. 26.

Abb. 4 Ibid. S. 81, Fig. 27.

Abb. 5 Misch, J., Rumpel, C., Die Kriegsverletzungen der Kiefer und der angrenzenden Weichteile. Berlin 1916, S.425, Fig. 478-480.

Abb. 6 Römer, O., Lickteig, A., Die Kriegsverletzungen der Kiefer. Strassburg i.E. 1917, S. 291, Abb. 52 .

Abb. 7 Ibid, S. 254, Abb. 22.

Abb. 8 Perthes, G., Borchers, E., Verletzungen und Krankheiten der Kiefer. Stuttgart 1932, S. 565, Abb. 218.

Abb. 9 Op. cit. sub 6, S.255, Abb. 24.

Abb.10 Op. cit. sub 5, S.462, Abb. 523.

Abb. 11 Op. cit. sub 6, S. 255, Abb. 23. 


\section{Summary}

From resection-prosthesis to free bone transplantation: Conditions to create a bone-transplantation concept for the lower jaw

The present paper examines the development of dental and surgical methods for the reconstruction of bone defects in the lower jaw. Starting with the resection-prosthesis we highlight important stages of bone transplantation in the lower jaw. Until 1914, actually no concept existed for reconstruction of continuity in the lower jaw. Only when dentists and surgeons started working together in the First World War, a bone-transplantation concept for the lower jaw could be elaborated.

\section{Résumé}

De la prothèse immédiate à la transplantation libre: Des conditions pour la réalisation des transplantations d'os à la mandibule

Le présent article analyse le développement des méthodes odontologiques et chirurgicales pour la reconstruction des défects d'os à la mandibule. Partant de la prothèse immédiate dans les résections du maxillaire inférieur on met en évidence les étapes importantes pour la transplantation à la mandibule. Jusqu'en 1914 on n'avait pas trouvé un concept réel pour reconstruire la mandibule. Seule la coopération entre dentistes et chirurgiens pendant la Première Guerre a rendu possible cette transplantation.

Dr. med. dent. Guido Sigron

Medzinhistorisches Institut der Universität

Rämistrasse 71

CH-8006 Zürich 